\title{
Financial Assessment of London Plan Policy 4A.2 by Probabilistic Inference and Influence Diagrams
}

\author{
Amin Hosseinian-Far, Elias Pimenidis, \\ Hamid Jahankhani, and D.C. Wijeyesekera \\ School of Computing, IT and Engineering, \\ University of East London \\ \{Amin, E.Pimenidis, Hamid.Jahankhani, Chitral\}@uel.ac.uk
}

\begin{abstract}
London Plan is the London mayor's Spatial Development Strategy. This strategic long-term plan comprises of proposals for different aspects of change within the London boundary. Furthermore, the proposals include chapters outlining adjustments in each facet. Policy 4A.2 reflects the Climate Change Mitigation scheme. Some consultations and research works have been performed to this point, but an extensive cost assessment has not been done. This paper reflects a financial assessment by means of Influence Diagrams based upon the London Plan policies 4A.X.
\end{abstract}

Keywords: London Plan, Climate Change Mitigation, Influence Diagrams, Probabilistic Inference, Analytica, Sustainability.

\section{Introduction}

There are various definitions for Sustainable City which is also known as eco-city. They generally denote to a city which tries to have the minimum energy, and food input and has the minimum $\mathrm{CO} 2$ emission, energy waste, general waste, methane and water pollution. The term was first introduced by Richard Register in 1987 as " $A n$ Ecocity is an ecologically healthy city" [1]. There are different plans for attaining the status of a sustainable city; they even vary from town to town and city to city. The United Kingdom is among the contributors to ecocity plans.

The aim of this paper is to demonstrate how influence diagrams can be used to do knowledge representation for a strategic, uncertain scenario. The case study selected is the London Plan Policy 4A.2. Section 1 discusses the key features of the London Plan relevant to this work; Section 2 outlines Influence Diagrams and the advantages of using them for this scenario. Finally the introduction to ID implementation is considered.

\section{London Plan}

The London Plan is the Mayor's Spatial Development Strategy, which covers different aspects of London's development; and a draft version of the plan has been out for public consultation since 2006 [2]. The section of the plan that this research 
focuses on is that of tackling the climate change and addressing sustainability analysis for London as a city. It is a strategic plan using a planning system and a set of aims and objectives relating to Energy Strategy and the control of climate change. The policies in the London Plan outline the proposals and schemes from London Transport strategic arrangement to the city Design. Climate Change is a sub division of the overall proposal.

\subsection{Climate Change Policy Area}

Climate Change as a sub division of the London Plan includes policies concerning different aspects of climate change from Air, Noise, Tackling Climate Change and even to Hazardous Substances. The Tackling Climate Change is the main concern of this research study. The policies involved are 4A.1 Tacking Climate Change, 4A.2 Mitigating Climate Change, and finally 4A.3 Sustainable Design and Construction. In spite of the categorization, these policies have overlapping areas between each other. The basis for the model which will be described in this work is Policy 4A.2, but some statistics and proposals will be borrowed from 4A.1 and 4A.3.

\subsection{Tackling Climate Change}

The main focus of this policy is on $60 \%$ carbon dioxide reduction in London by 2050 relatively to the 1990 base. Comparatively, 15\%, 20\%, 25\%, and 30\% reduction targets have been set for 2010, 2015, 2020, and 2025 [2]. According to the plan draft new Building Development regulations are needed to be introduced; not only the new built properties should be regulated, but also the existing houses and properties should adapt to the criteria accordingly. According to Day, Ogumka, \& Jones 20\% out of the $60 \%$ reduction can be gained through the use of renewable energies [3]. Foreman Roberts research debates that the $20 \%$ out of $60 \%$ solely by renewables is not achievable [4]. Despite of all discussions, the cost assessment will be performed by only considering the policies published by the Greater London Authority (GLA). There are 147 case studies by further plan analysis, as approved by the London mayor. One of the main concerns of the plan is that of the use of Combined Heat and Power (CHP). CHP utilization would the most cost effective approach in the London Plan [5].

The other two policies focus on more thorough details of the London Climate Change route. According to research conducted by London South Bank University the impact assessment of the approach shown in Figure 1 should prove suitable to assess the general perspective of the London Plan climate change policies:

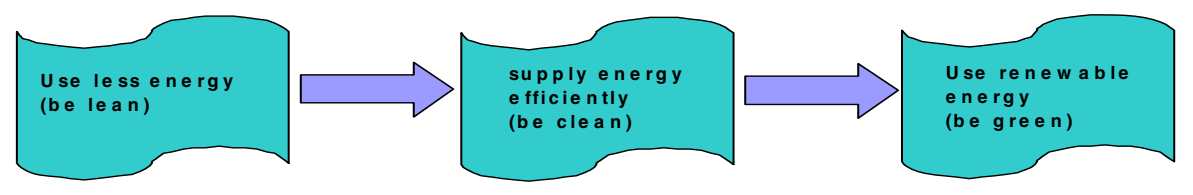

Fig. 1. Climate Change policy in three main phase [6] 
According to the same research, the level of $\mathrm{CO} 2$ emission savings based on Energy Efficiency is higher than what could be expected by the use of renewable energy, as shown in table 1 below.

Table 1. Energy Efficiency and Renewables savings in the London Plan

\begin{tabular}{|l|c|c|c|}
\hline & \multicolumn{2}{|c|}{$\begin{array}{c}\text { Savings to date from 113 energy } \\
\text { statements }\end{array}$} & $\begin{array}{c}\text { Savings to date }- \text { scaled for 350 } \\
\text { developments }\end{array}$ \\
\hline & tonnes $\mathrm{CO}_{2} / \mathrm{y}$ & $\%$ & tonnes $\mathrm{CO}_{2} / \mathrm{y}$ \\
\hline $\mathrm{EE}$ & 111,492 & 21.3 & 345,329 \\
\hline $\mathrm{RE}$ & 24,039 & 5.8 & 74,457 \\
\hline Total & 135,528 & 25.8 & 419,777 \\
\hline
\end{tabular}

One of the debates relating to the London Plan (LP) is that of the targets set by the government. The government has set $60 \% \mathrm{CO} 2$ reduction by 2050 ; below with the specific figure of $60 \%$ being quite unclear as to what the government envisages. Fig. 2 illustrates the most predominant understanding and it this viewpoint that the work presented here adopts:

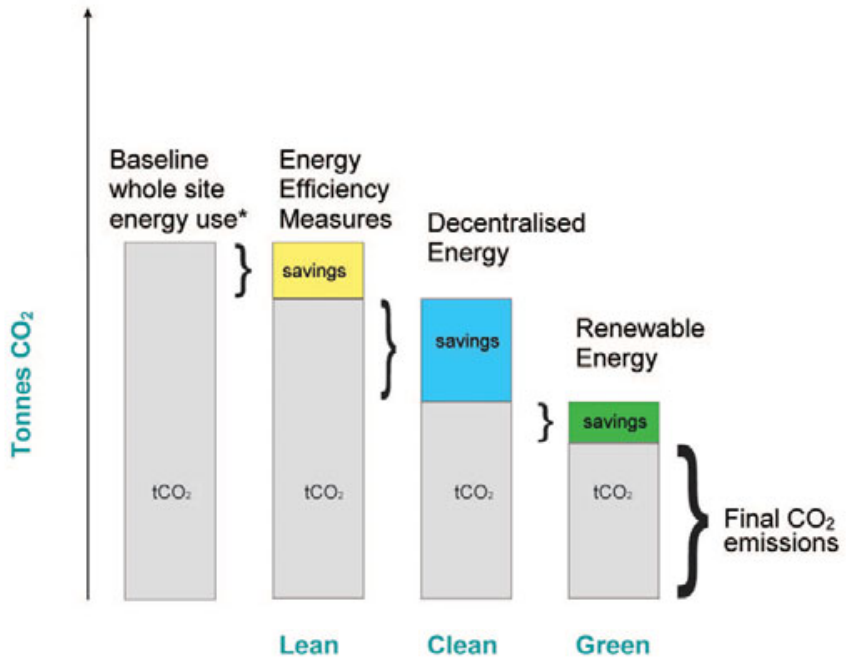

Fig. 2. Calculation of energy/carbon dioxide savings [7]

Energy Efficiency is the first phase according to the plan and also based on the LSBU research data is shown as having the highest reduction. The use of Combined Heat and Power (CHP) which is also called cogeneration refers to generation of electricity as well as heating the development. Renewable energy systems do not play a major role in the reduction process, but the introduction of more efficient renewables could yield an advantage. The application of the plan on 260 approved developments has revealed the following results: 


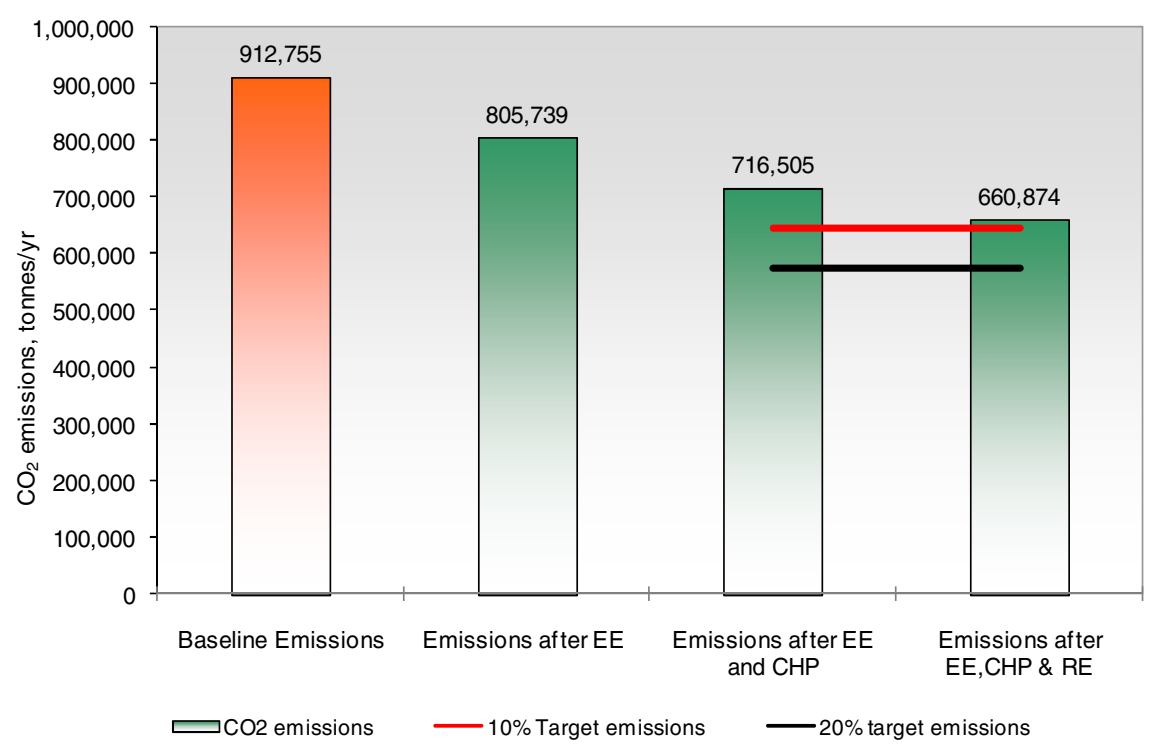

Fig. 3. Savings and approaches [6]

\section{Influence Diagrams}

An Influence Diagram (ID) is a network visualization for probabilistic models [8]. It also includes decision analysis and can be easily converted to decision trees [9]. Howard and Matheson initially introduced the concept in 1981 and according to them Influence Diagrams can be considered as a bridge between qualitative and quantitative analysis [10]. The concept of ID has had substantial influence on Artificial Intelligence (AI) and has made AI more mature [11].

An influence diagram consists of nodes and arcs. The nodes and arcs form a visual network [12]. The nodes may be of different types: Variables, Constants, Decision Nodes, Chance Node, and Objective; although there are other naming conventions for the ID nodes. They can also be classified as deterministic or probabilistic nodes. The Arcs specify the relationship and dependencies of the variables [13]. Definition of each node determines an associated mathematical expression for each node.

Influence Diagrams are a proper tool for decision making and analyzing a scenario under uncertainty [14]. Applying ID to London Plan policy analysis is an appropriate approach as the London Plan involves some uncertainty points. Furthermore Influence Diagrams can be furthermore broken down and illustrate more details. In complex situations such as the London Plan, the boundaries and level of details are in the hands of the analyst and can even be further refined later.

A Well-Formed Influence Diagram (WFID) is an ID which is not only well visualized, but also includes well-defined mathematical expressions for the nodes [15]. In addition WFIDs can be evaluated using reversal and removal approaches. This can be used for complex inferential and probabilistic scenarios [16]. 
In terms of categorization and classification of the subject, Influence Diagrams are classified under 'Bayesian Updating, Certainty Theory and Fuzzy Logic' category (Fig. 4).

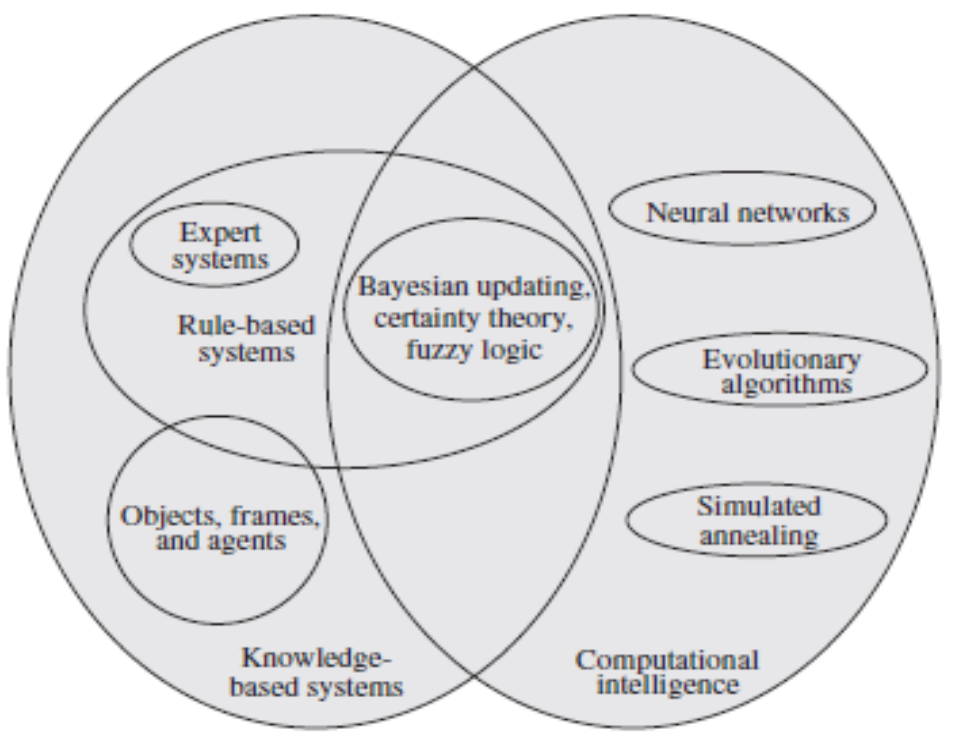

Fig. 4. Artificial Intelligence Classification [17]

\section{Implementation}

\subsection{Indicators}

A set of indicators for the London Plan monitoring phase is introduced, but the list does not cover the 4A.X policies. Therefore the indicators and variables used in the model are derived from the LP qualitative and quantitative statements within the boundary of this study.

\subsection{Model Creation}

The Integrated Development Environment (IDE) used for this research is called Analytica, many academic and industrial users of the software review Analytica as one of the most powerful applications for modeling using Influence Diagrams; even though other modeling environments can be used.

There are various recommendations for the starting point of influence diagrams. A systemic approach is to create the systems map first, and based on the systems map the influence diagram can be created. By using the systems map, the areas of the analysis and boundaries of the system under investigation will be determined [18]. In addition the influential parts of the research would be easier to find. A tentative and general systems map for this research is outlined in Fig. 5: 


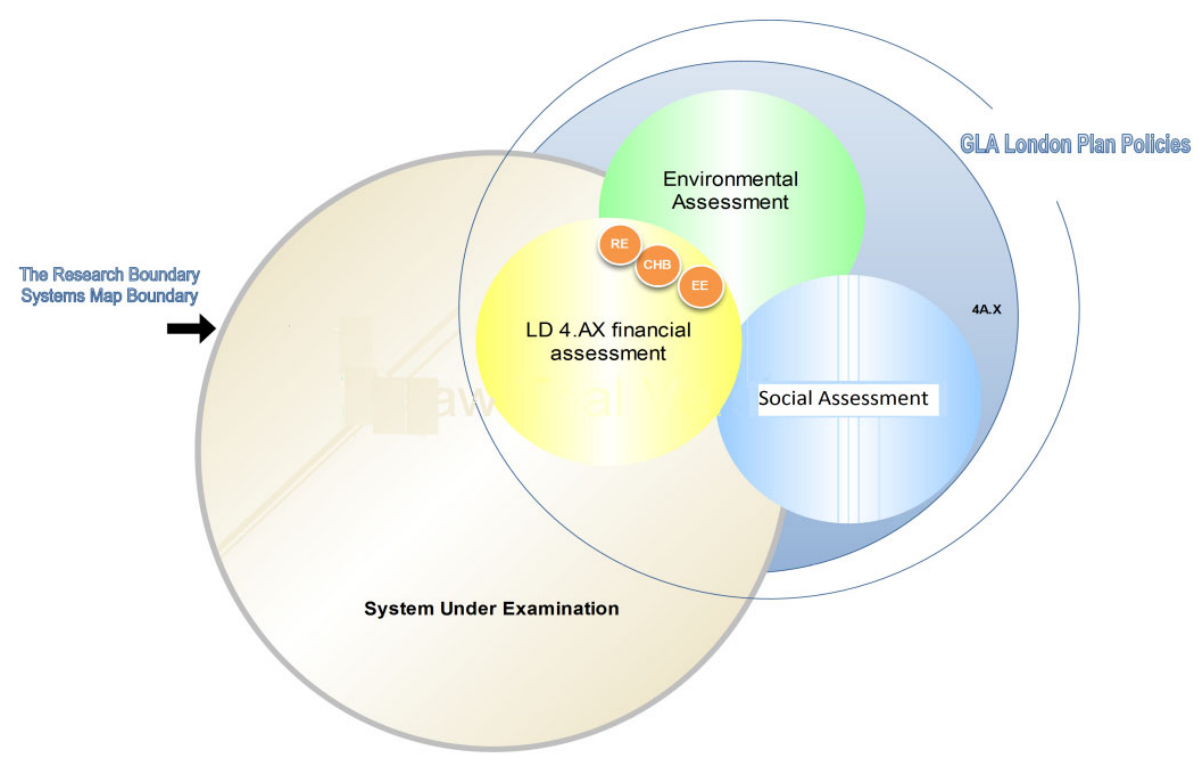

Fig. 5. Systems Map for London Plan Policy 4A.2

The systems map illustrated above draws a boundary for the system. The Environmental and Social assessments of the LD policy 4A.X overlap with these two domains. The economic or financial assessment domain is fully within the boundary. The use of renewable energies, energy efficiency procedures, and also less use of energy here outlined as CHB are the sub components which are located in the overlapping area of the three domains of sustainability. Based on this diagram a general visual representation of policy4A.2 analysis is demonstrated using a Probabilistic Network in Fig. 6:

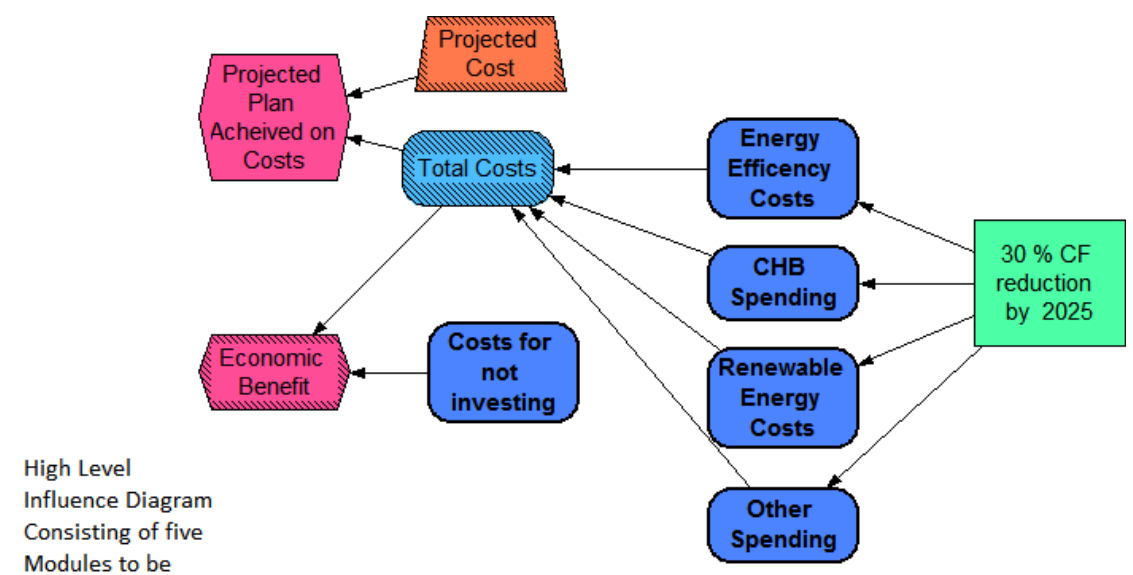

further expanded

Fig. 6. High level Influence Diagram for London Plan Policy 4.A.2 
The above general model consists of five modules which will be further detailed in the context of this work. The decision node or policy node is '30\% Carbon Footprint (CF) reduction by $2025^{\prime}$. This is one of the nodes that do not include a mathematical expression while it only demonstrates an information passing process, rather than actual calculability.

The above decision node includes:

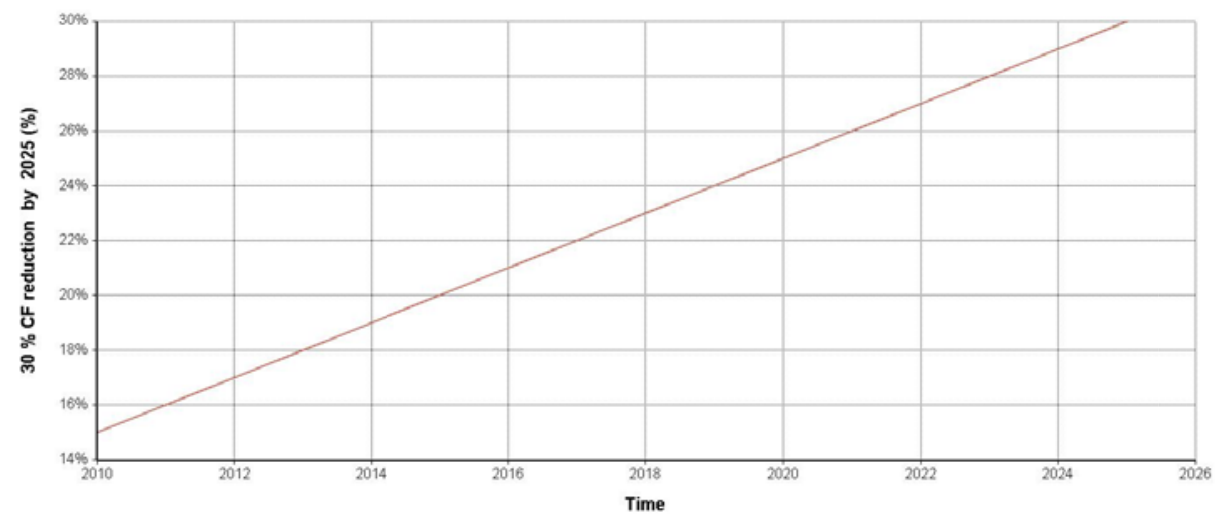

Fig. 7. Decision node: $30 \%$ CF Reduction by 2025 considering the 1990's base

The linearity of the output is due to the input being the contents of a table of data rather than a non linear equation. The probability distribution of the cost for not investing is as shown below:

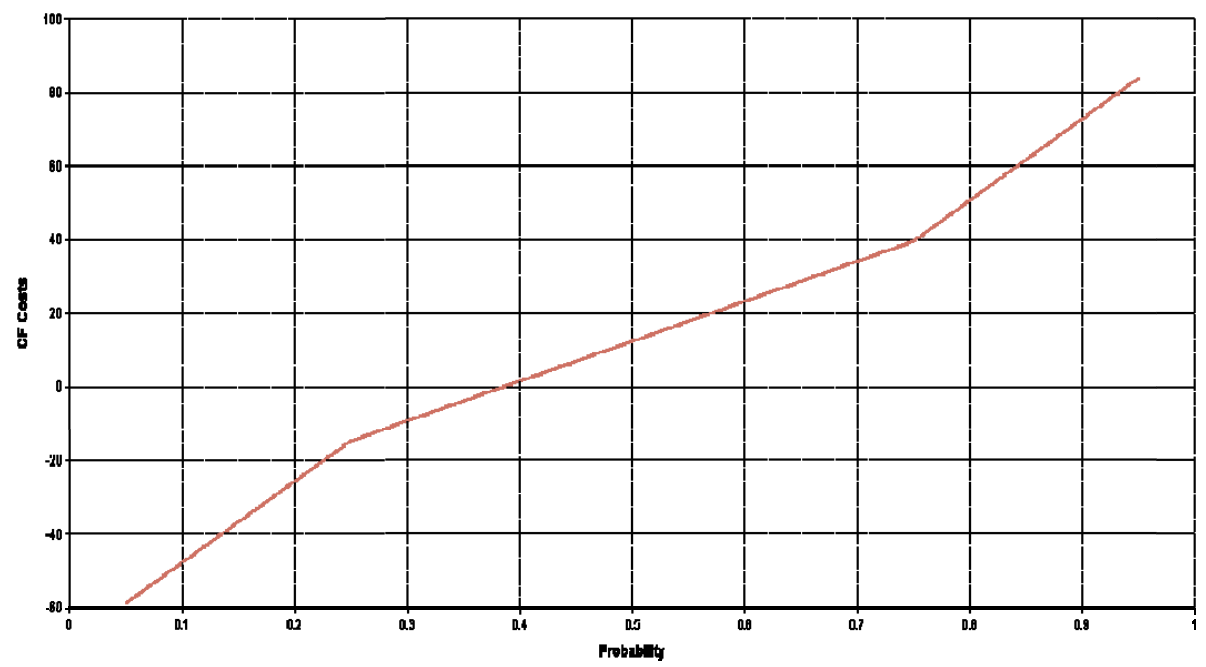

Fig. 8. Cost of Carbon Footprint by 2030, a tentative output illustrating different possible degrees of membership and the CF cost 


\section{If Projected_cost $>$ Total_Costs, Then True, Else False}

The above influence diagram and its modules and sub diagrams are to be created after this initial generalized model. But the reasons for choosing the influence diagrams for this type of policy modeling are due to its advantages. Influence diagrams have many advantages over decision trees. In decision trees adding nodes and generally expanding the model with more details requires to add nodes as child within the tree and that adds nodes to the whole diagram exponentially. Another issue with that is articulating conditional independence relationships within a decision tree would be easier said than done. Some levels of expressions within influence diagrams cannot be developed using decision trees [10].

A key question here would be that of how to evaluate the ID created. There are different naming used for different types of ID. A regular ID is the one with decision nodes in order, one after the other within a specified path. The not-forgetting ID is the one that information for previous decision nodes is also available for other decision nodes. A well-formed influence diagram or abbreviated as WFID is an influence diagram with well defined mathematical and probability expressions within itself. There are different ways in order to evaluate an influence diagram. The evaluation technique for WFID would be the reversal and removal operations in order to reach the starting node. For regular and other forms of influence diagrams there is the chance for conversion of the ID into a Bayesian network, although these two are quite the same. Converting an ID into a Bayesian Network is a straightforward task, as both have many similarities. All the nodes within the ID should be converted into chance nodes. The reason for that is in a Bayesian network the nodes are representing a probability distribution and no exact node should be defined. For conversion of the decision nodes into probability nodes or chance nodes, the newly defined chance node should have an even distribution:

$$
\forall\langle\langle d i \in \operatorname{dom}(d i), P(\langle d i| \pi d i)=1 /|\operatorname{dom}(d i)|
$$

Where di $\in D$ corresponds to decisions to be made.

It is once simulated for a probability less than $50 \%$ (can be tentatively named true chance), and once for a probability over 50\% (which alternatively can be named as the false chance). Then it can be simulated as Bayesian network and evaluated using the Bayesian algorithms. This technique is called Coppers reduction which was initially introduced by Cooper in 1988. There are other algorithms and techniques which will enable us to evaluate ID e.g. Shachter and Peot's Algorithm, Zhang's Algorithm, Xiang and Ye algorithm and etc [19].

The completed ID for policy 4A.2 can be evaluated by means of an extensive comparison between the result of the ID and a scientific project management approach. The evaluation methods discussed above can also be utilized for that purpose.

\section{Conclusion and Further Work}

It appears that the concept of Influence Diagrams is compatible with the nature of decision making challenges paused by the London Plan and in particular the financial viability of the use of Renewable Energy Systems to partly meet LP's targets. 
Completion of the above ID would be a proper means for evaluating the financial domain of the London Plan 4A.x policy. Apart from completing the ID, evaluation of the ID is essential.

Policy making for tackling climate change, energy crisis and a probable catastrophe has been urged by many governments. Some have started it already using various technical and scientific research studies. However, some policies are still at political level and there is no proper research work to allow for the validation of such plans.

According to the United Nations MDG indicators datasets, China and United States are the most $\mathrm{CO} 2$ polluters. Political and cultural similarities between the UK and the USA confirm that the next step for the use this climate change policy modelling approach would be the US cities with the highest CO2 emission [20].

Similar policies can be applied to them. Finally, use of other renewable energy systems would not cause any amendments to the general structure of the research using Influence Diagrams.

\section{References}

1. Register, R.: Ecocity Berkeley, Building Cities for a Healthy Future. North Atlantic Books, US (1987)

2. Friends of Earth: The Mayor's London Plan Briefing Report. Friends of Earth (FEO) Website (2006)

3. Day, A.R., Ogumka, P., Jones, P.G., Dunsdon, A.: The Use of the Planning System to Encourage Low Carbon Energy Technologies in Buildings. Renewable Energy 34(9), 2016-2021 (2009)

4. Roberts, F.: Renewables and the London Plan. British Council for Offices (March 2007)

5. Cibse: Small-scale combined heat and power for building. Guide 176 in Cibse website (2011)

6. Day, T.: Renewable Energy in the City. In: Advances in Cumputing and Technology Conference, London (2011)

7. Greater London Authority: The London Plan, Spatial Development Strategy for Greater London, Consolidated with Alterations since 2004. London Mayor's website (February 2008)

8. Shachter, R.D.: Probabilistic Inference and Influence Diagrams. Stanford University, Stanford (1987)

9. Ronald, A.H., James, E.M.: Influence Diagrams. Decision Analysis 2(3), 127-143 (2005)

10. Howard, R.A., Matheson, J.E.: Influence diagrams (1981). In: Howard, R.A., Matheson, J.E. (eds.) Readings on the Principles and Applications of Decision Analysis, vol. 2. Strategic Decisions Group, Menlo Park (1984)

11. Boutilier, C.: The Influence of Influence Diagrams on Artificial Intelligence. Decision Analysis 2(2), 229-231 (2005)

12. Shachter, R.D.: Model Building with Belief Networks and Influence Diagram. In: Edwards, W., Ralph, F., Miles, J., Winterfeldt, D.V. (eds.) Advances in Decision Analysis: From Foundations to Applications, pp. 177-201. Cambridge University Press, Cambridge (2007)

13. Influence Diagrams, http: / / www. Iumina.com/technology/influence-diagrams / 
14. Liao, W., Ji, W.: Efficient Non-myopic Value-of-information Computation for Influence Diagrams. International Journal of Approximate Reasoning 49(2), 436-450 (2008)

15. Sage, A.P.: Systems Engineering. Wiley Series in System Engineering (1992)

16. Shachter, R.: Evaluating Influence Diagrams. Operations Research 34(6), 871-882 (1986)

17. Hopgood, A.A.: Intelligent Systems for Engineers and Scientists, 2nd edn. CRC Press, New York (2001)

18. Open University: Influence Diagrams, http: //openlearn.open.ac.uk/mod/oucontent/ view. php? id=397869\&section=1.9.4

19. Crowley, M.: Evaluating Influence Diagrams: Where we've been and where we're going. Unpublished Literatrue review (2004)

20. Daily Green: Top 20 CO2-Polluting U.S. Cities and Suburbs (2011), retrieved April 2011, from Daily Green: http://www.thedailygreen.com/environmentalnews/latest/carbon-emissions-47041804 